\title{
Feasibility of skin carotenoids as a biomarker of vegetable intake among Mexican-Origin children in a community setting: A cross-sectional pilot study
}

\author{
Alberto L. Aguilera*1,2, Christine P. Stewart ${ }^{1}$, Adela de la Torre ${ }^{2,3}$, Lucia L. Kaiser ${ }^{1}$ \\ ${ }^{1}$ Department of Nutrition, University of California, Davis, United States \\ ${ }^{2}$ Center for Transnational Health, University of California at Davis, Davis, United States \\ ${ }^{3}$ Chicana/o Studies Department, University of California at Davis, Davis, United States
}

Received: January 30, 2017

Accepted: March 22, 2017

Online Published: September 19, 2017

DOI: $10.5430 /$ jer.v4n1p1

URL: https://doi.org/10.5430/jer.v4n1p1

\begin{abstract}
Background and purpose: Studies in adults and children have demonstrated a strong correlation of skin carotenoids with fruit and vegetable $(\mathrm{F} / \mathrm{V})$ intake. There are few studies testing the usefulness of this methodology in a community setting. This study aimed to determine the feasibility of resonance Raman spectroscopy (RRS) skin carotenoid intensity scores in young children to assess F/V intake.

Methods: A cross-sectional study design was used to capture the RRS skin carotenoid intensity scores using the Pharmanex BioPhotonic Scanner. The RRS skin carotenoid intensity score ranges from 0 to 70,000+ with increasing scores indicating a higher carotenoid concentration. Children's dietary intake of F/V was assessed within one-month period using a 26-item food frequency questionnaire (FFQ) and multiple pass 24-hour dietary recalls (24HDRs) administered to the parent of the child. Participants were 51 Mexican-origin children (ages 3-8 years) living in two rural California school districts during July-August 2014. RRS skin carotenoid intensity scores; frequency of fruit and vegetable intake from FFQ; daily servings of fruit and vegetables and carotenoid intake from 24HDR. Spearman correlation was used to determine the correlation between RRS skin carotenoid intensity scores and average 24HDR F/V intake and FFQ F/V intake.

Results: The results of the study demonstrate a significant positive correlation between skin carotenoid levels and parent-reported 24HDR vegetable consumption ( $r=.41, p=.003)$ but not with 24HDR fruit, FFQ fruit, or FFQ vegetable consumption.

Conclusion: The use of skin carotenoid measurements may be more indicative of short-term vegetable consumption than overall fruit and vegetable or fruit-only consumption in this population of Mexican-origin children.
\end{abstract}

Key Words: Carotenoids, Children, Mexican, Fruits and vegetables, Resonance Raman spectroscopy

\section{INTRODUCTION}

There are a variety of tools that are used for dietary assessment, including food frequency questionnaires (FFQ), 24hour dietary recalls (24HDR), and food records. ${ }^{[1]}$ Though the FFQ and 24HDR tools pose less participant burden than food records, a major drawback of these two methods is their reliance on participant recall, which can lead to over-reporting of healthy foods and underreporting of unhealthy foods. ${ }^{[2]}$ Dietary assessment is particularly challenging in children, where studies have demonstrated mixed States.

*Correspondence: Alberto L. Aguilera; Email: alaguilera@ucdavis.edu; Address: Department of Nutrition, University of California, Davis, United 
results on the accuracy and reliability of child-reported or parent/caretaker-reported instruments. ${ }^{[3]}$ Various factors such as age, gender, weight status, and social desirability affect participant reporting and introduce bias when using 24HDR and FFQ methods. ${ }^{[2]}$ A systematic review of selfreported and parent-reported dietary assessments in children, compared to doubly labeled water, concluded that selfreported dietary recalls in children younger than nine years are unreliable. The use of parental or caretaker multiplepass dietary recalls for children ages 4-9 years old is considered to provide an accurate measurement. ${ }^{[4]}$ However, FFQs and 24HDRs rely on individual recall, which can introduce bias. Given these considerations, alternatives to FFQs and 24HDRs that can capture fruit and vegetable intake quickly, accurately, and objectively would prove to be valuable tools for research.

The Institute of Medicine considers serum carotenoids as the gold standard biomarker of fruit and vegetable (F/V) consumption since they are only found in high concentrations in these sources. ${ }^{[5]}$ The use of serum carotenoids as a biomarker for fruit and vegetable consumption has been validated in adults. ${ }^{[6]}$ Furthermore, a systematic review demonstrated the usefulness of carotenoids as a biomarker for fruit and vegetable intake. ${ }^{[7]}$ Vegetables rich in carotenoids increase the level of carotenoids present in blood and in the skin compared to vegetables less rich in carotenoids. ${ }^{[8]}$ However, the use of serum carotenoids presents many challenges including cost; participant burden; individual factors (e.g. bioavailability); limitations from nutrition databases; and practicality in community settings. In the past decade, a novel method of measuring carotenoid levels in the skin using resonance Raman spectroscopy (RRS) was developed. ${ }^{[9,10]}$ The ability of RRS to measure carotenoid levels has been validated against serum and skin biopsy carotenoid levels in an adult population with various skin melanin levels. ${ }^{[10]}$ Studies have demonstrated positive correlation between skin carotenoid levels and $\mathrm{F} / \mathrm{V}$ consumption $(r=.61, p<.001)$ in adults ages 18-30 years and children ages 5-17 years $\left(R^{2}=0.18\right.$, $p<.05){ }^{[11,12]}$ In children ages $9-12$, RRS skin carotenoids are correlated with plasma carotenoids $(r=.62, p<.001)$ and total carotenoids as reported from an FFQ $(r=.40, p$ $<.0001){ }^{[13]}$ However, there are no previous studies that have compared the usefulness of this methodology in a rural community with a large Mexican immigrant population.

The Niños Sanos, Familia Sana (NSFS) study is a threeyear, multi-component intervention study aimed at preventing childhood obesity in Mexican-origin children ages 3 to 8 years in rural California. ${ }^{[14]}$ A secondary aim of the study is to increase fruit and vegetable consumption among children. Based on a subsample from the NSFS study, this study examines feasibility of RRS skin carotenoid measurements in comparison to an FFQ and 24HDR in young Mexicanorigin children within a rural, largely-immigrant, community setting. It is important to evaluate the usefulness of new methods in groups with different cultural food habits and choices.

\section{Methods}

\subsection{Study design and population}

This study uses a cross-sectional design to compare fruit and vegetable consumption assessed by FFQ and 24HDR to RSS skin carotenoid levels, all of which were collected within the same month period during summer 2014. The study took place in a rural community in California. The Institutional Review Board at University of California, Davs approved the study protocol. Parents or legal guardians signed informed consent forms in their preferred language (Spanish or English).

\subsection{Study population}

The study sample included children of Mexican heritage in rural California. Children were eligible to be included in this study if 1) their families were enrolled in the larger NSFS study; 2) they were between ages $4-8$ years; and 3) their parents consented to the collection of three dietary recalls, FFQs and RSS measures. ${ }^{[14]}$ If families had enrolled more than one child in the study, only one child closest to the ages 4-5 years was selected to capture information before they enter the school system. In cases where a household had more than one eligible child between 4-5 years, the younger child was selected to participate in the study. Parents provided informed consent but assent from children in this age group was not required.

\subsection{Sample size}

The sample size for this study was computed using a lowest expected correlation of 0.35 between the FFQ and RRS skin carotenoid concentrations based on previous studies in adults and children. ${ }^{[10,12,15-17]}$ A sample of 50 was estimated to detect a correlation of at least 0.35 with approximately $80 \%$ power (alpha $=0.05$, two-tailed). To allow for incomplete data, we recruited 79 participants.

\subsection{Dietary assessment}

Bilingual, trained research assistants collected three 24HDR measurements from study participants. The 24HDR was parent-reported (in-person and via phone call) using the multiple-pass diet recall method for the diet of children for two weekdays and one weekend day were collected within one month. ${ }^{[1]}$ The first diet recall was in-person and subsequent recalls were conducted over the phone. Multiple-pass 
diet recall methodology is deemed to be an appropriate approach to collecting accurate dietary information from individuals by reducing potential recall bias. The first dietary recall was scheduled in-person at a local office. The same research assistants collected subsequent dietary recalls over the phone using a standardized protocol. Participants were given a \$10 gift card for completion of all three dietary recalls. The Nutrition Data System for Research (NDSR) visual portion handbook (Version 2012) assisted the parents in estimating servings of all foods. The $24 \mathrm{HDR}$ intake data were analyzed using the NDSR software (version 2012, Nutrition Coordinating Center, University of Minnesota, Minneapolis, $\mathrm{MN}){ }^{[18]}$ The resulting dietary intakes were an average of all days collected. Using definitions set by the 2015-2020 Dietary Guidelines for Americans, a serving of fruit was 1/2 cup of cut fruit (e.g. a medium banana or apple) or 1/2 cup fruit juice, and a serving of vegetable was 1 cup of raw leafy vegetables, $1 / 2$ cup of cooked or raw vegetables, or $1 / 2$ cup of vegetable juice. ${ }^{[19]}$ During the dietary recall, parents were asked if the child consumed any supplements or vitamins. In addition to servings of fruit and vegetables, the NDSR database also provided estimates for carotenoid intakes.

The researchers administered a 26 item FFQ with a 5category Likert response scale $(1=$ never or almost never; 2 $=1$ time or less a week; $3=2-3$ times a week; $4=1$ time or less a day; and $5=$ more than 1 time a day) to ask parents about their children's intake of fruit and vegetables and other foods over the previous 30 days. ${ }^{[20]}$ The FFQ was collected within a month after first dietary recall administered. The FFQ F/V consumption was calculated by creating a sum of 7 items (fresh fruit, canned fruit, vegetable soup, other cooked vegetables, salsa, lettuce or cabbage, and other vegetables) to produce a score (range: 7-35). FFQ fruit consisted of 2 items (fresh fruit and canned fruit) with range of 2-10. The FFQ Veg variable consisted of 5 items (vegetable soup, other cooked vegetables, salsa, lettuce or cabbage, and other vegetables) to with a range of 5 to 25 . The FFQ instrument used in this study has demonstrated construct validity and internal reliability in this population. ${ }^{[20]}$ In another study, children's F/V consumption, as assessed by this FFQ, was correlated with household inventory of F/V. ${ }^{[21]}$

The RRS skin carotenoid skin carotenoid levels of the children were measured during the first visit using the Pharmanex BioPhotonic Scanner (S2 Everest Edition, Pharmanex, Provo, UT, 2007). The scanner uses RRS to measure carotenoid levels at a point between the distal and proximal palm of the right hand. Skin carotenoid intensity scores in the palm area do not differ by skin tone and considered appropriate measurement location. ${ }^{[15]}$ The scanner emits $473 \mathrm{~nm}$ beam of blue light that is then bent to $510 \mathrm{~nm}$ green light. Carotenoids

Published by Sciedu Press are the only compounds known to bend a $473 \mathrm{~nm}$ light to $510 \mathrm{~nm} .{ }^{[22]}$ The RRS skin carotenoid measurement results in a continuous intensity score ranging from 0 to $70,000+$ that indicates increasing carotenoids present in the skin with increasing intensity score. The palm area was exposed to the scanner for 90 seconds. ${ }^{[12,17]}$ Two readings were collected. If the differences in measurements were greater than 5,000, a third measurement was collected. The final RRS skin carotenoid score was an average of the two closest measurements.

Trained researchers and staff collected anthropometric measurements of the children during home visits and at a school site using the NSFS study protocol from the Anthropometric Standardization Reference Manual. ${ }^{[23]}$ Children were asked to remove their shoes and wear only light clothing. The child's height was measured in centimeters to the nearest 0.1 cm using a portable stadiometer (Model 213, Seca GmbH \& Co. KG, Hamburg, Germany). The weight of each child was measured in kilograms to the closest $0.05 \mathrm{~kg}$ using a digital scale (Model 874, Seca GmbH \& Co. KG, Hamburg, Germany). Child BMI z-scores were calculated using the CDC growth charts. ${ }^{[24]}$ Detailed description of anthropometric methodology is described elsewhere. ${ }^{[14]}$

\subsection{Statistical analysis}

All data analyses were completed using SAS software (version 9.3, SAS Institute Inc., Cary, NC, USA). ${ }^{[25]}$ Data were visually inspected using scatterplots to examine linearity. Ttests and chi-square were used to examine differences in RRS skin carotenoid scores, FFQ F/V, and 24HDR F/V across subgroups (child age, child gender, BMI z-score). A Spearman correlation analysis among all study variables was performed to determine bivariate unadjusted relationships. Linear regressions were conducted with FFQ or 24HDR fruit and vegetable intake variables, controlling for child's age, gender, BMI z-score. The level of significance was set at $\mathrm{p}<0.05$ for all statistical analyses.

\section{RESULTS}

Out of 79 recruited, the final sample included 51 children. Twenty-eight participants did not complete the study due to lack of interest or being unavailable for follow-up within the desired time period. Fifty-one participants completed at least one dietary recall. Of those who completed the first recall, 31 completed a second dietary recall, and 13 completed a third dietary recall. A skin carotenoid measurement and FFQ was collected from all 51 children who completed the study. Out of all dietary recalls collected, three were removed from the analysis due to caloric intake that was considered too low (below 800 kilocalories/day). Skin carotenoid measurements, 
FFQ, and dietary recalls were all completed within the same month. Summary characteristics of the sample are shown in Table 1 . There were no statistically significant differences in demographic characteristics between those who completed the study and those who did not. There were no statistically significant differences in RRS skin carotenoid scores by gender (see Table 2). However, overweight and obese children had significantly lower RRS skin carotenoid intensity scores compared to normal weight children.

No significant differences in the consumption of recall reported fruits were observed by age, sex, and weight status for citrus juice, fruit juice, fruit (excluding citrus fruit), avocado, fried fruits, and fruit-based savory snacks (Data Not Shown). Although not significant, normal weight children had 0.18 servings of citrus juice compared to 0.04 servings in overweight children $(p=.0619)$. Girls had 0.10 servings of starchy vegetables (not including potatoes) compared to 0.01 servings in boys ( $p=.0493$ ). No other significant differences were observed among 24HDR vegetable intake by age, sex, or weight status.

Table 1. Baseline characteristics of participants in a study to determine if RRS skin carotenoids are useful among Mexican-origin children in rural California as a biomarker of $\mathrm{F} / \mathrm{V}$ intake $(\mathrm{n}=51)$

\begin{tabular}{ll}
\hline Characteristics & Mean \pm SD or \% \\
\hline Child age (years) & $5.8 \pm 1.5$ \\
Child sex (male) & $56 \%(\mathrm{n}=29)$ \\
Child BMI-z score & $0.93 \pm 1.02$ \\
Maternal age (years) & $35.7 \pm 6.5$ \\
Maternal education (years), $\mathrm{n}=50$ & $8.9 \pm 4.0$ \\
Maternal BMI (kg/m $\left.{ }^{2}\right), \mathrm{n}=39$ & $30.8 \pm 4.5$ \\
Household income (US\$/year), $\mathrm{n}=46$ & $19835 \pm 1556$ \\
\hline
\end{tabular}

Table 2. Bivariate comparison of resonance Raman spectroscopy (RRS) skin carotenoids; food frequency questionnaire fruit and vegetable intake (FFQ F/V); and 24-hour dietary recall fruit and vegetable intake (24HDR F/V) in a study to determine if RRS skin carotenoids are useful among Mexican-origin children in rural California as a biomarker of F/V intake $(n=51)$

\begin{tabular}{|c|c|c|c|c|c|}
\hline Characteristic & $\mathbf{N}(\%)$ & $\begin{array}{l}\text { Mean RRS } \\
\text { Score (SD) } \\
\end{array}$ & $\begin{array}{l}\text { Mean FFQ } \\
\text { F/V } \mathbf{V}^{\ddagger} \text { (SD) } \\
\end{array}$ & $\begin{array}{l}\text { Mean 24HDR } \\
\text { F/V servings (SD) }\end{array}$ & $\begin{array}{l}\text { Mean } 24 H D R \\
\text { Daily caloric intake }\end{array}$ \\
\hline \multicolumn{6}{|l|}{ Ages (years) } \\
\hline 3 to 5 & $16(30.0 \%)$ & $26,817(9,021)$ & $17.75(3.7)$ & $5.8(4.6)$ & $1,432(507)$ \\
\hline 6 to 8 & $35(70.0 \%)$ & $29,010(12,968)$ & $16.9(3.6)$ & $4.2(3.1)$ & $1,369(433)$ \\
\hline \multicolumn{6}{|l|}{ Sex } \\
\hline Male & $29(56.8 \%)$ & $26,336(9,832)$ & $16.9(3.9)$ & $4.8(4.1)$ & $1,441(545)$ \\
\hline Female & $22(43.2 \%)$ & $31,760(13,834)$ & $17.4(2.7)$ & $4.6(2.9)$ & $1,285(233)$ \\
\hline \multicolumn{6}{|l|}{ Weight Status } \\
\hline Normal $(<85 \%$ ile $)$ & $19(44.1 \%)$ & $32,522(15,829)$ & $18.2(3.7)$ & $4.6(3.3)$ & $1,192(265)$ \\
\hline Overweight/obese ( $\geq 85 \%$ ile) & $24(55.9 \%)$ & $25,721(7,021)^{*}$ & $16.1(3.2)^{*}$ & $5.1(4.3)$ & $1,536(535)^{* * *}$ \\
\hline
\end{tabular}

\section{Skin carotenoid comparison to FFQ and 24HDR F/V consumption}

The RRS carotenoid intensity scores were not correlated with FFQ total fruit and vegetable; FFQ fruit, and FFQ vegetable intakes. The 24HDR vegetable intake $(r=.414, p=.003)$, but not total F/V ( $r=.188, p=\mathrm{NS})$ or fruit intake $(r=.104, p$ = NS), was significantly correlated with RRS skin carotenoid intensity scores. Total carotenoid intakes, as reported by the 24HDR, were not correlated with RRS skin carotenoid scores $(r=.251, p=.079)$. When controlling for child's age, gender, and BMI z-score, 24HDR F/V, 24HDR Vegetable, to- tal 24HDR carotenoid intake, 24HDR lycopene, and 24HDR lutein + zeaxanthin were found to be significant (see Table 3).

\section{DISCUSSION}

The results of this study suggest that noninvasive skin carotenoids can be a viable measure of vegetable consumption in Mexican-origin children in a rural community setting. Specifically, RRS skin carotenoid intensity scores may be more indicative of vegetable intake than fruit intakes in this population. Another study in children $(n=50)$ found that 
reported vegetable intake more strongly correlated $(r=0.32,0.21, p=.02) .{ }^{[12]}$

$p=.0001)$ with RRS skin carotenoid than fruit intake $(r=$

Table 3. Linear regression of resonance Raman spectroscopy skin carotenoid intensity score (RRS) with fruit and vegetable $(\mathrm{F} / \mathrm{V})$ intake from food frequency questionnaire (FFQ) and 24-hour dietary recall (24HDR) controlling for child's age, gender, BMI z-score in a study to determine if RRS skin carotenoids are useful among Mexican-origin children in rural California as a biomarker of $\mathrm{F} / \mathrm{V}$ intake $(\mathrm{n}=51)$

\begin{tabular}{|c|c|c|c|}
\hline & RRS $R^{2}$ & RRS ß (SE) & $p$-value \\
\hline \multicolumn{4}{|l|}{ Frequency of consumption } \\
\hline $\mathrm{FFQ} F / \mathrm{V}^{\dagger}$ & 0.27 & $754(532)$ & .165 \\
\hline FFQ Fruit ${ }^{\ddagger}$ & 0.30 & $3136(1770)$ & .085 \\
\hline FFQ Vegetable & 0.25 & $713(656)$ & .285 \\
\hline \multicolumn{4}{|l|}{ Servings } \\
\hline 24HDR F/V & 0.30 & $941(429)$ & .034 \\
\hline 24HDR Fruit & 0.24 & $812(639)$ & .212 \\
\hline 24HDR Vegetable & 0.32 & $2135(831)$ & .014 \\
\hline \multicolumn{4}{|l|}{ Total amount, mcg } \\
\hline 24HDR total carotenoids & 0.32 & $0.48(0.18)$ & .014 \\
\hline $24 \mathrm{HDR} \beta$ carotene & 0.24 & $0.69(0.58)$ & .230 \\
\hline 24HDR $\alpha$ carotene & 0.22 & $1.12(1.55)$ & .450 \\
\hline 24HDR lycopene & 0.31 & $0.57(0.24)$ & .023 \\
\hline 24HDR cryptoxanthin & 0.25 & $11.1(7.5)$ & .152 \\
\hline 24HDR lutein + zeaxanthin & 0.32 & $10.1(3.9)$ & .015 \\
\hline
\end{tabular}

A possible explanation for the weak correlations between the RRS skin carotenoid scores and FFQ F/V index is due to the types of fruits and vegetables consumed by these children. The subgroups of fruit and vegetable in this sample that contributed the most to total intake were non-citrus fruits (including bananas and apples) and other vegetables (including vegetables in salads, soups, stews, stir-fry, and similar mixed dishes), which possess fewer concentrations of carotenoids. These children consumed relatively low amounts of darkgreen vegetables, deep-yellow vegetables, and tomatoes, which are carotenoid-rich sources. Household food purchase data obtained using scanners at the point of purchase also provide evidence of this: the top ten fruits and vegetables purchased in this population were (in descending order): bananas, apples, tomatoes, carrots, avocados, jalapeno peppers, cucumbers, iceberg lettuces, cilantro, and strawberries (Blinded for Review: unpublished data, September 2015). This is similar to national data of most commonly purchased and consumed fruits and vegetables, which are apples, bananas, iceberg lettuce, onions, citrus (fruit and juices), and tomato products. ${ }^{[26,27]}$

Published by Sciedu Press
Except tomatoes and carrots, fruits and vegetables most commonly purchased do not contain high concentrations of carotenoids. ${ }^{[5]}$ If children were consuming more fruits than vegetables, this would increase the total fruit and vegetable intake but would not necessarily result in higher skin carotenoid scores. This observation may explain why there was no correlation between fruit intakes (by either the FFQ or 24HDR) with skin carotenoid intensity scores. Another reason that may explain the differences observed between 24HDR and FFQ is the items on each instrument. The FFQ used in this study is limited to only one fruit (all fresh fruit) item and seven vegetable items while the 24HDR does not have this same limitation. The FFQ may have been too limited to capture the variety of fruits and vegetables consumed by the population. Also, this FFQ only is designed to measure the frequency of consumption, whereas the 24HDR yields an estimate of amounts consumed as well.

There were challenges in collecting three 24-hour dietary recalls from participants in this population. Participant availability and dispersion across small, rural communities hin- 
dered obtaining all dietary recalls required to do standard analysis using this method. This posed an issue with the dietary recall, which requires multiple contact points, whereas the RRS skin carotenoid intensity scores and an FFQ measurements can be completed at one contact point with no loss of participation. Furthermore, the skin carotenoid intensity scores can be easily captured in a centralized location such as a school campus or community center and do not require the presence of the parent/guardian, as FFQ or dietary recalls do. Given these challenges, using RRS skin cartenoid intensity scores to measure vegetable intake in this population is may be a viable option. Although there is an initial lease or purchase of the appropriate equipment for RRS skin carotenoid measurements, they do not require further software, specific technical training, or data analysis processing costs as FFQ or 24-hour dietary recalls.

This study has several strengths and limitations. The study population includes children of Mexican-origin from rural California, a difficult to reach population. The current study is one of the few studies to implement the use of skin carotenoid measurements in a rural Mexican immigrant community setting with young children. Two dietary assessment instruments (FFQ and 24HDR) were used to compare skin carotenoid intensity scores as a measure of fruit and vegetable intake. Conversely, the study did not compare skin carotenoids to serum or skin carotenoid levels, measures that would have been challenging to obtain in this communitybased study setting. The FFQ in this study only used limited number of items to capture fruit and vegetable intake frequency and may have biased reporting. Since this study included a small sample size from a difficult to reach population, the results of this study may not be generalized to other Mexican-origin populations. Due to challenges in this hard-to-reach population, it would be important to over recruit due to a higher dropout rate. Lastly, three 24HDR were not obtained from all participants. The lack of three complete dietary recalls from all participants likely introduces a bias in the food group and nutrient estimates. Although two 24HDR are deemed appropriate for larger population studies such as the NHANES, three complete dietary recalls are best for comparisons of nutrition assessment tools and individual nutrient intake. Therefore, the findings from this study should be interpreted with these limitations. However, it is important to note the challenges in obtaining three 24-hour dietary recalls from populations similar to the one in this study and the value of RRS skin carotenoid measurements provides when fruit and vegetable intake is the variable of interest.

\section{Implications}

RRS skin carotenoid score may be a useful biomarker for vegetable intake, but not total fruit and vegetable intake, in young Mexican-origin children in a community setting. There is potential to use RRS skin carotenoid scores as a quick and easy measure for interventions aiming to increase vegetable intake in young children. Future studies should further investigate how carotenoid levels change throughout the year to determine the effects of seasonality on dietary patterns in this population as well as how to effectively translate these findings to enhance fruit and vegetable consumption in these high risk communities. A cost-analysis of RRS skin carotenoid intensity scores versus other methods would also be beneficial in future studies.

\section{CONFlicts OF INTEREST Disclosure}

The authors declare that they have no competing interests.

\section{REFERENCES}

[1] Monsen ER, Van Horn L. Research: Successful Approaches: American Dietetic Associati; 2007.

[2] Livingstone MBE, Robson PJ. Measurement of dietary intake in children. Proceedings of the Nutrition Society. 2000; 59(02): 279-93. https://doi.org/10.1017/S0029665100000318

[3] Burrows T, Truby H, Morgan P, et al. A comparison and validation of child versus parent reporting of children's energy intake using food frequency questionnaires versus food records: who's an accurate reporter? Clinical Nutrition. 2013; 32(4): 613-8. PMid:23206381 https://doi.org/10.1016/j.clnu.2012.11.006

[4] Burrows TL, Martin RJ, Collins CE. A Systematic Review of the Validity of Dietary Assessment Methods in Children when Compared with the Method of Doubly Labeled Water. Journal of the American Dietetic Association. 2010; 110(10): 1501-10. https: //doi.org/10.1016/j.jada.2010.07.008
[5] Food Nutrition Board IoM. Dietary Reference Intakes for Vitamin C, Vitamin E, Selenium, and Carotenoids. National Academy of Sciences. 2000.

[6] Resnicow K, Odom E, Wang T, et al. Validation of Three Food Frequency Questionnaires and 24-Hour Recalls with Serum Carotenoid Levels in a Sample of African-American Adults. American Journal of Epidemiology. 2000; 152(11): 1072-80. https://doi .org/10 $.1093 / a j e / 152.11 .1072$

[7] Burrows TL, Williams R, Rollo M, et al. Plasma carotenoid levels as biomarkers of dietary carotenoid consumption: A systematic review of the validation studies. Journal of Nutrition \& Intermediary Metabolism. 2015; 2(1-2): 15-64. https : //doi.org/10.1016/j. jnim. 2015.05.001

[8] Pezdirc K, Hutchesson MJ, Williams RL, et al. Consuming HighCarotenoid Fruit and Vegetables Influences Skin Yellowness and Plasma Carotenoids in Young Women: A Single-Blind Randomized 
Crossover Trial. Journal of the Academy of Nutrition and Dietetics. 2016; 116(8): 1257-65. https://doi.org/10.1016/j.jand.2 016.03 .012

[9] Hammond BR, Wooten BR. Resonance Raman spectroscopic measurement of carotenoids in the skin and retina. Journal of Biomedical Optics. 2005; 10(5): 054002. https://doi.org/10.1117/1.21 16767

[10] Mayne ST, Cartmel B, Scarmo S, et al. Noninvasive assessment of dermal carotenoids as a biomarker of fruit and vegetable intake. The American Journal of Clinical Nutrition. 2010; 92(4): 794-800. https://doi.org/10.3945/ajcn.2010.29707

[11] Jahns L, Johnson LK, Mayne ST, et al. Skin and plasma carotenoid response to a provided intervention diet high in vegetables and fruit: uptake and depletion kinetics. The American Journal of Clinical Nutrition. 2014; 100(3): 930-7. https://doi.org/10.3945/ajcn .114 .086900

[12] Aguilar SS, Wengreen HJ, Dew J. Skin Carotenoid Response to a High-Carotenoid Juice in Children: A Randomized Clinical Trial. Journal of the Academy of Nutrition and Dietetics. 2015; 115(11): 1771-8. https://doi.org/10.1016/j.jand.2015.06.011

[13] Nguyen LM, Scherr RE, Linnell JD, et al. Evaluating the relationship between plasma and skin carotenoids and reported dietary intake in elementary school children to assess fruit and vegetable intake. Archives of Biochemistry and Biophysics. 2015. https: //doi.org/10.1016/j.abb.2015.02.015

[14] de la Torre A, Sadeghi B, Green R, et al. Ninos Sanos, Familia Sana: Mexican immigrant study protocol for a multifaceted CBPR intervention to combat childhood obesity in two rural California towns. BMC Public Health. 2013; 13(1): 1033. https://doi.org/10.1 186/1471-2458-13-1033

[15] Scarmo S, Cartmel B, Lin H, et al. Single v. multiple measures of skin carotenoids by resonance Raman spectroscopy as a biomarker of usual carotenoid status. British Journal of Nutrition. 2013; FirstView: 1-7. https ://doi.org/10.1017/S000711451200582X

[16] Scarmo S, Henebery K, Peracchio H, et al. Skin carotenoid status measured by resonance Raman spectroscopy as a biomarker of fruit and vegetable intake in preschool children. European Journal of Clinical Nutrition. 2012; 66(5): 555-60. https://doi.org/10.1038/ ejcn.2012.31

[17] Wengreen HJ, Madden GJ, Aguilar SS, et al. Incentivizing children's fruit and vegetable consumption: Results of a United States pilot study of the Food Dudes program. Journal of Nutrition Education and Behavior. 2013; 45(1): 54-9. PMid:23178042 https: //doi.org/10.1016/j.jneb.2012.06.001

[18] NDSR. 2012 ed. Minneapolis, MN: Nutrition Coordinating Center; 2012.

[19] U.S. Department of Agriculture, U.S. Department of Health and Human Services. Dietary Guidelines for Americans, 2015. 8th ed2015.

[20] Kaiser LL, Aguilera AL, Horowitz M, et al. Correlates of food patterns in young Latino children at high risk of obesity. Public Health Nutr. 2015: 1-9. https://doi.org/10.1017/S1368980014003 309

[21] Kaiser L, Martin A, Metz D, et al. Food insecurity prominent among low-income California Latinos. California Agriculture. 2004; 58(1): 18-23. https://doi.org/10.3733/ca.v058n01p18

[22] Ermakov IV, Gellermann W. Validation model for Raman based skin carotenoid detection. Archives of Biochemistry and Biophysics. 2010; 504(1): 40-9. https://doi.org/10.1016/j.abb.2010.0 7.023

[23] Lohan T, Roche A, Martorell R. Anthropometric Standardization Reference Manual: Champaign: Human Kinetics Publishers; 1988.

[24] Kuczmarski R, Ogden C, Grummer-Strawn L, et al. CDC growth charts: United States. Adv Data. 2000; 1-27. PMid:11183293

[25] SAS. 9.3 ed. Cary, NC, USA: SAS Institute Inc.; 2011.

[26] Nielsen SJ, Rossen LM, Harris DM, et al. Fruit and vegetable consumption of US youth, 2009-2010. NCHS data brief. 2014; 156: $1-8$.

[27] Food Availability and Consumption [Internet]. United States Department of Agriculture. 2012. Available from: http://www.ers.usda.gov/data-products/ag-and-foo d-statistics-charting-the-essentials/food-availab ility-and-consumption . aspx\#.U4-WDJRg6K4 